\title{
Single-molecule Imaging of Shaker Ion Channel Stabilized by Detergent and Amphiphilic Copolymer
}

\author{
Dmitry Bagrov $^{1}$, Julia Kacher ${ }^{1}$, Grigory Gluhov ${ }^{2}$, Olga Sokolova ${ }^{3}$ and Konstantin Shaitan ${ }^{1}$ \\ ${ }^{1}$ Moscow State University named after M.V.Lomonosov, Moscow, Moskva, Russia, ${ }^{2}$ Moscow State \\ University named after M.V.Lomonosov, Sochi, Moskva, Russia, ${ }^{3}$ Lomonosov Moscow State University, \\ Faculty of Biology, Moscow, Moskva, Russia
}

Membrane proteins maintain their native structure only if embedded into a lipid bilayer. This is true for the voltage-gated potassium ion channels, which play a fundamental role in neuron functioning. Here we studied Shaker ion channel, a tetrameric protein with $\sim 300 \mathrm{kDa}$ molecular weight. Being a membrane protein, Shaker requires special treatment for extraction and solubilization. The traditional approach to this problem is the application of detergents. Despite the wide variety of the available detergents, usually, they do not preserve the membrane protein structure as efficiently as the natural lipids. Recently alternative approaches emerged; they exploit either nanodiscs, lipid particles stabilized by the membrane scaffold protein, or SMALPs, lipid particles stabilized by styrene-maleic acid copolymer (SMA). These approaches have some advantages over the detergent-based structure stabilization [1], [2]. Here we isolated Shaker protein from HEK293 cell membranes using two techniques - the detergent-based (using CHAPS detergent) and the SMALP-based (using SMA copolymer). We used dynamic light scattering (DLS), atomic force microscopy (AFM) and transmission electron microscopy (TEM) to compare the size of Shaker molecules in the obtained preparations. Shaker was expressed in HEK293 cells, and the membrane fraction was obtained by cell lysis and the subsequent centrifugation. Either $1 \%$ CHAPS or $2.5 \%$ SMA was added to the membrane fraction to solubilize the membrane proteins. Shaker was purified using affinity chromatography. DLS measurements were carried out using Zetasizer Nano ZS (Malvern). TEM images were obtained using JEM-2100 microscope (Jeol), AFM images were obtained using Solver PRO microscope (NT-MDT). DLS showed that the hydrodynamic diameter of the particles was $20 \pm 6 \mathrm{~nm}$ in both studied samples (number-based distribution). This value was far larger than the typical $\sim 10 \mathrm{~nm}$ size of the "empty" SMALP nanoparticles [3]. Shaker molecules appeared as globules in the AFM (Figure 1) and TEM (Figure 2) images. In AFM images, the mean height of the molecules was 3.6 $\pm 1.0 \mathrm{~nm}$ for the Shaker+XIRAN sample and $4.4 \pm 1.2 \mathrm{~nm}$ for the Shaker+CHAPS sample. The particles with height $\mathrm{h}<2.5$ $\mathrm{nm}$ were discarded during processing. The lateral size of the particles was larger in the AFM images $(30 \pm 10 \mathrm{~nm}$ for the Shaker+XIRAN sample and $44 \pm 6 \mathrm{~nm}$ for the Shaker+CHAPS sample) than in the TEM images $(12 \pm 3 \mathrm{~nm}$ for the Shaker+XIRAN sample and $13 \pm 4 \mathrm{~nm}$ for the Shaker+CHAPS sample) due to the AFM tip broadening. According to the AFM and DLS data, the SMALP particles with incorporated Shaker molecules were larger than the previously described "empty" SMALPs. This fact contrasts with the previously described measurements of the archaeal photoreceptor/transducer complex NpSRII/NpHtrII157 [3, 4]. 

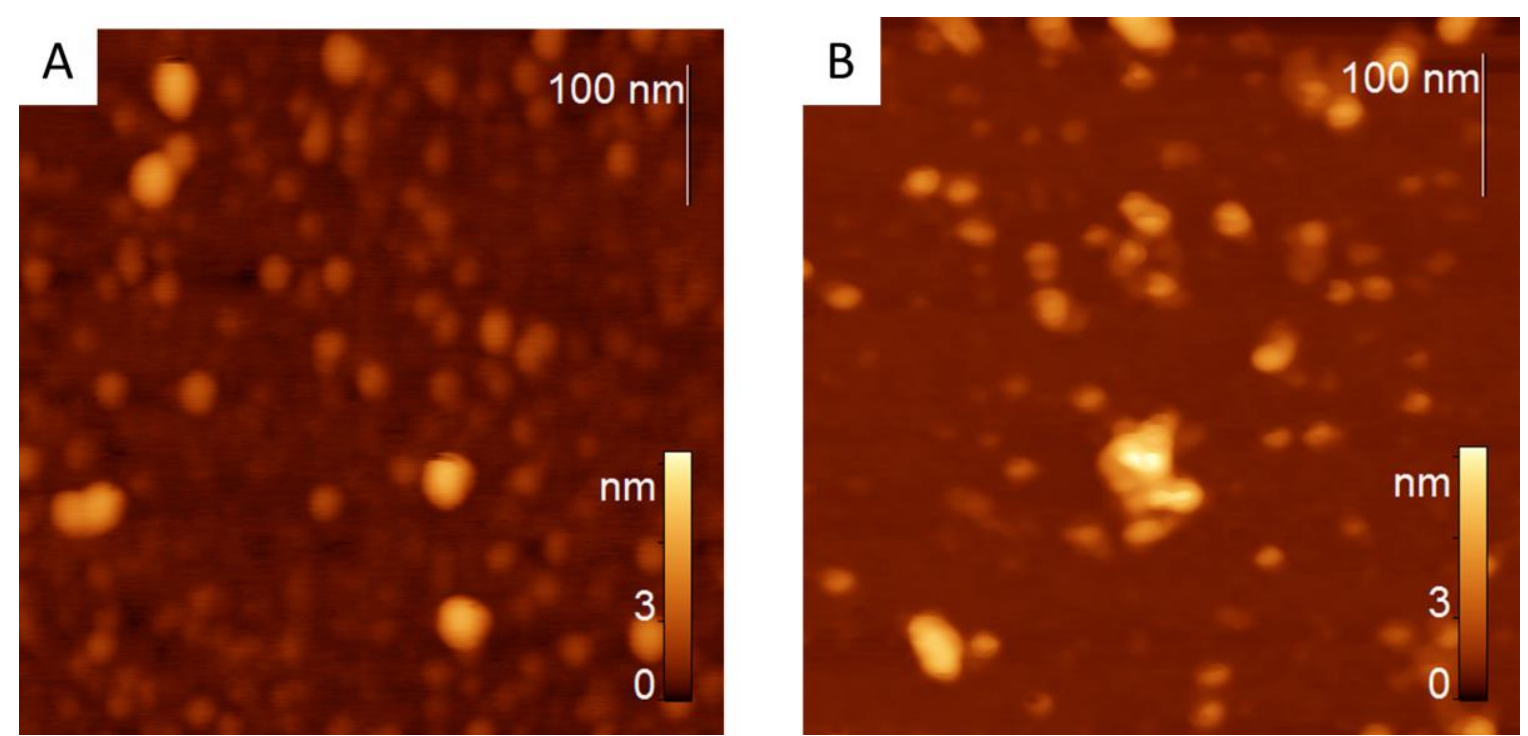

Figure 1. AFM images of the Shaker ion channel stabilized by CHAPS (A) and XIRAN (B)
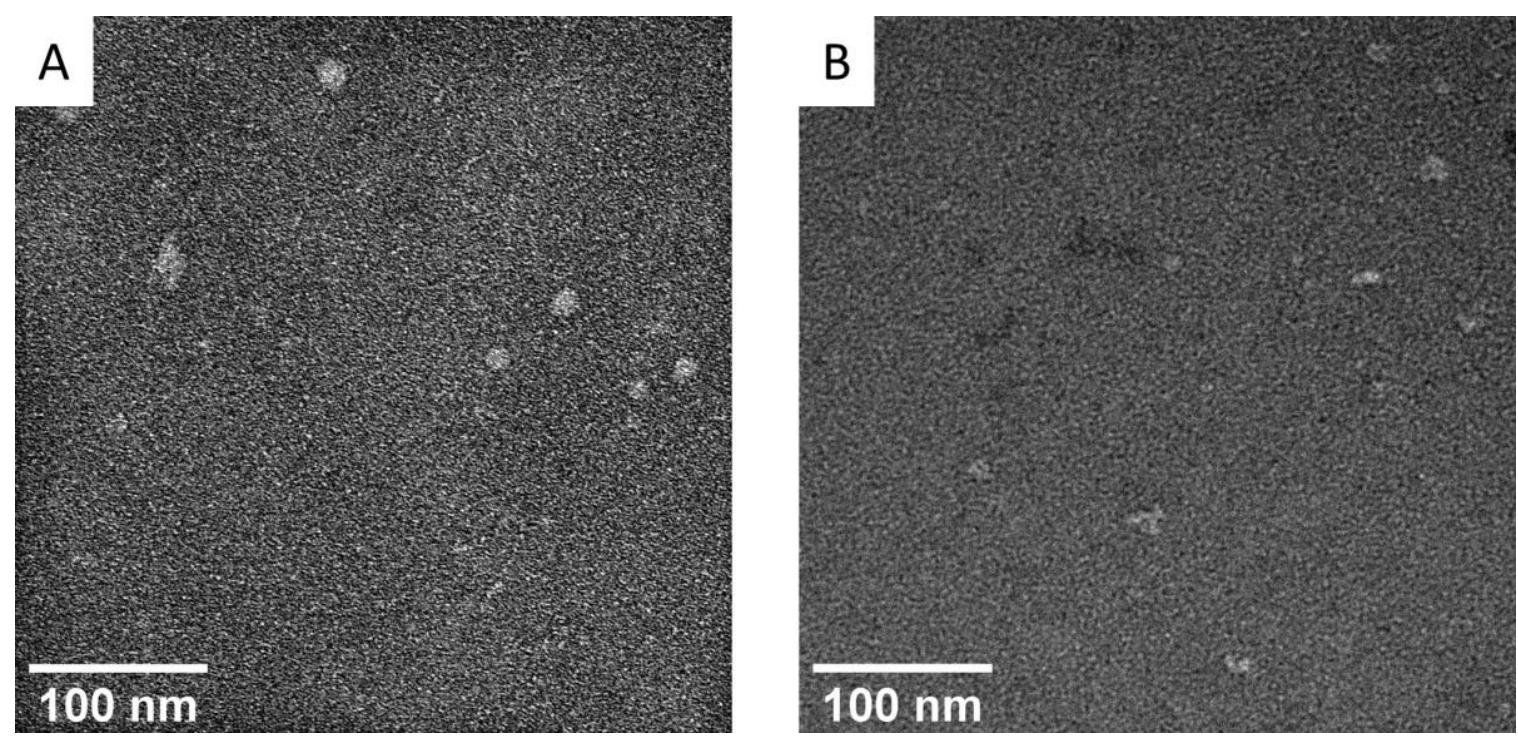

Figure 2. TEM images of the Shaker ion channel stabilized by CHAPS (A) and XIRAN (B)

\section{References}

[1] N. Voskoboynikova et al., RSC Adv., vol. 7, no. 81, pp. 51324-51334, 2017. [2] M. G. Karlova et al., Chem. Phys. Lipids, vol. 219, pp. 50-57, Mar. 2019. [3] D. V. Bagrov et al., Biophysics (Oxf)., vol. 61, no. 6, pp. 942-949, Nov. 2016. [4] The work was supported by RFBR, project 18-50412045. The research was carried out using the quipment purchased on account of the Lomonosov MSU Development Program. Electron microscopy was performed on the "3D-EMC" instrument cluster of Moscow State University (supported by Ministry of science and higher education of Russian Federation, unique identifier RFMEFI61919X0014). 